\title{
On Dimensionality Loss in FDD Massive MIMO Systems
}

\author{
Zhiyuan Jiang, Student member, IEEE, Sheng Zhou, Member, IEEE, and Zhisheng Niu, Fellow, IEEE \\ Tsinghua National Laboratory for Information Science and Technology, \\ Department of Electronic Engineering, Tsinghua University, Beijing, 100084, China \\ jiang-zy10@mails.tsinghua.edu.cn, \{sheng.zhou,niuzhs\}@ tsinghua.edu.cn
}

\begin{abstract}
Dimensionality loss is defined as the channel estimation overhead, which results in a loss of time-frequency resources in pilot-assisted wireless systems. In this paper, the scaling result of dimensionality loss, i.e., the scaling factor, in frequency-division-duplex (FDD) massive multiple-inputmultiple-output(MIMO) downlinks is derived. The scaling factor determines the amount of channel estimation overhead, and thus is vital to understand the downlink throughput in FDD massive MIMO systems. Moreover, the transmit diversity of the downlink channel is also derived. In the simulations, we adopt a geometry-based stochastic channel model to validate our analysis. The impact of several assumptions made in our analysis is also investigated.
\end{abstract}

\section{INTRODUCTION}

Massive multiple-input-multiple-output (MIMO) systems, along with millimeter-wave transmissions and ultra cell densification have been widely recognized as the big three technologies in $5 \mathrm{G}$ wireless communication systems [1]. By deploying a large excess number of antennas at base station (BS) side, the massive MIMO system not only significantly improves the performance of the local cell, including cell throughput, radiated energy efficiency and simplicity of media-accesscontrol (MAC) layer design [2] [3], but also shows promising advantages in integrating with small-cell technologies due to the large excess spatial dimensions for inter-tier interference cancelation.

For any application scenarios, one of the fundamental premisses of massive MIMO systems is that the BS has channel state information at transmitter (CSIT). Otherwise, the spatial multiplexing gain would be lost, and the system is reduced to a single-user MIMO system in terms of throughput. Moreover, the BS would not be capable of exploiting the additional spatial modes to transmit to intended users while also suppressing the interference to other cells or tiers. In view of the vital importance of CSIT, to obtain it, cellular systems adopt pilot-assisted schemes, which can be categorized by open-loop and closed-loop schemes. In antenna-array calibrated timedivision-duplex (TDD) systems, the open-loop scheme is used where the BS obtains the CSIT by uplink channel training thanks to the hard-earned reciprocal uplink-downlink channels. The reason for venturing the sophisticated antenna-array calibration is the tremendous dimensionality loss reduction by the open-loop scheme, wherein the dimensionality loss, which is defined as the amount of time-frequency resources in a fading

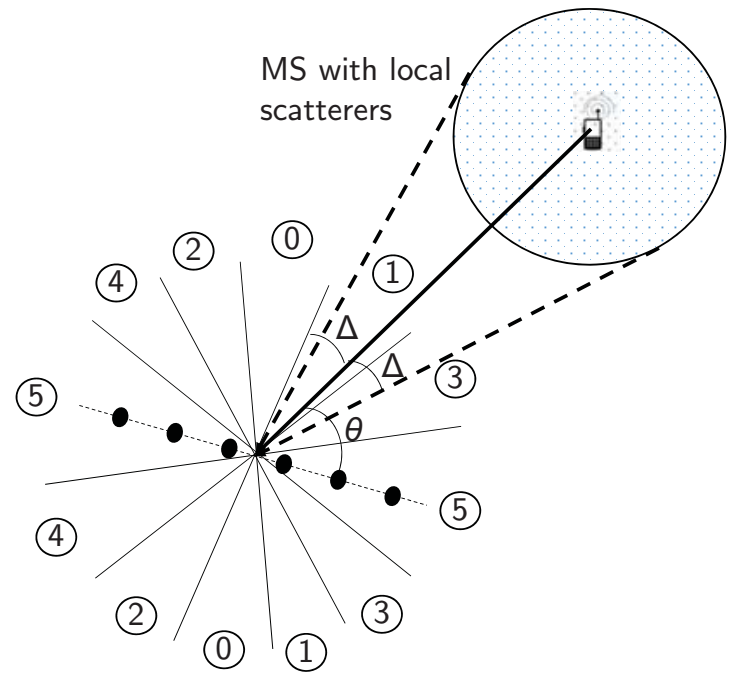

Fig. 1. User-signal angular spread introduced by local scatters with directionof-arrival (DoA) $\theta$ and angular spread $\Delta$. The number of bins denotes the angular resolution of BS antennas.

block $^{1}$ dedicated to channel estimations, scales only with the number of concurrent users, irrelevant with the number of BS antennas. Therefore, dramatically increasing the number of BS antennas benefits the system with the limitation solely related to antenna-design or processing complexity considerations. However, in FDD or uncalibrated TDD massive MIMO systems, a closed-loop channel estimation scheme is mandatory, which means that the BS must send downlink training signals and listen to user CSIT feedback, whereby the dimensionality loss scales with the number of BS antennas, making the dimensionality loss unacceptable with a large antenna-array. Therefore, it is generally assumed that the massive MIMO system is only practical with a calibrated TDD mode [2], due to the prohibitively large dimensionality loss otherwise. However, this assumption significantly restricts the use of massive MIMO, and some frequency bands are dedicated to the FDD mode. Therefore, it is of great importance and urgency to understand the nature of dimensionality loss in FDD massive MIMO systems.

Recently, it has been shown that the dimensionality loss with closed-loop channel estimation does not necessarily scale with

\footnotetext{
${ }^{1}$ In this paper, we assume block fading channels.
} 
the number of BS antennas in spatially correlated channels, but rather with the effective rank (neglecting extremely small singular values) of the channel correlation matrices (CCMs) [4]-[6]. Efficient training and CSI feedback schemes exploiting spatial correlations have been proposed [6]. The spatial channel correlation is in fact ubiquitous in cellular systems, given the fact that it is directly related to the angular power spectral (APS) of received signals, see Fig. 1, and that the APS shows distinct angular power concentration, in particular, the APS follows Laplacian distribution approximately shown by real measurements [7]. However, the connection between the angular power concentration and the dimensionality loss has been scantly treated, i.e., a qualitative characterization of the dimensionality loss given the signal APS is needed. This work aims to answer this question and also investigates the tradeoff between the dimensionality loss and the number of downlink transmit diversity, which is closely related to the degree-offreedom (DoF) of the downlink broadcast channel. In Section II, we describe the system model and present our main results. In Section III, we use a geometry-based stochastic channel model (GSCM) to validate our results. We conclude our work in Section IV.

Notations : Throughout the paper, we use boldface uppercase letters, boldface lowercase letters and lowercase letters to designate matrices, column vectors and scalars, respectively. $\boldsymbol{X}^{\dagger}$ denotes the complex conjugate transpose of matrix $\boldsymbol{X}$. $x_{i}$ denotes the $i$-th element of vector $\boldsymbol{x} \cdot \mathcal{C N}(\boldsymbol{\mu}, \boldsymbol{\Sigma})$ denotes a circularly symmetric complex Gaussian random vector of mean $\boldsymbol{\mu}$ and covariance matrix $\boldsymbol{\Sigma}$.

\section{System Model And Main Results}

Consider a downlink broadcast channel, where an $M$ antenna BS serves $N$ single-antenna users ${ }^{2}$, and the receive signal of user $n$ is

$$
y_{n}=\boldsymbol{h}_{n}^{\dagger} \boldsymbol{x}+n_{n}
$$

where $\boldsymbol{h}_{n}$ is an $M$-dimensional channel vector, $\boldsymbol{x}$ is the downlink transmit signals, and $\boldsymbol{n}$ denotes the Gaussian additive noise. The downlink channel matrix is denoted as $\boldsymbol{H}=$ $\left[\boldsymbol{h}_{1}, \boldsymbol{h}_{2}, \ldots, \boldsymbol{h}_{N}\right]^{\dagger}$. The CCM of the channel of user $n$ is

$$
\boldsymbol{R}_{n}=\mathbb{E}\left[\boldsymbol{h}_{n} \boldsymbol{h}_{n}^{\dagger}\right]
$$

By the Karhunen-Loeve (KL) representation,

$$
\boldsymbol{h}_{n}=\boldsymbol{R}_{n}^{\frac{1}{2}} \boldsymbol{z}_{n},
$$

where it is prescribed that $\boldsymbol{z}_{n} \sim \mathcal{C N}\left(\mathbf{0}, \boldsymbol{I}_{M}\right)$. The user channel vectors are mutually independent due to the separability of users in space. Furthermore, denote the singular-valuedecomposition (SVD) of the CCM as

$$
\boldsymbol{R}_{n}=\boldsymbol{U}_{n} \boldsymbol{\Sigma}_{n} \boldsymbol{U}_{n}^{\dagger}
$$

and $\boldsymbol{\Sigma}_{n}=\operatorname{diag}\left[\lambda_{1}^{(n)}, \lambda_{2}^{(n)}, \ldots, \lambda_{M}^{(n)}\right]$. It is worthwhile to stress that in this work, we assume the BS and the users have perfect

\footnotetext{
${ }^{2}$ Note that our results can be directly generalized to systems with multipleantenna users.
}

knowledge of the CCMs. This assumption can be justified in the sense that CCMs usually vary at a much lower rate than instantaneous CSIT, e.g., seconds to tens of seconds for CCMs whereas milliseconds for instantaneous CSIT, such that they can be estimated efficiently. It is instrumental for both the BS and users to know the CCMs, in order to reduce dimensionality loss [4].

From (3), we can illustrate the dimensionality loss scaling property qualitatively. When $\boldsymbol{h}_{n}$ is independently identically distributed (i.i.d.), i.e., $\boldsymbol{R}_{n}=\boldsymbol{I}_{M}$, assuming unit-norm entries, the dimensionality loss scales with $M$, i.e., the dimensionality scaling factor is $M$, which is the number of unknown variables if we consider the channel estimation process in analogy to solving linear-equations, ignoring the channel noise for the moment. Each linear-equation corresponds to one downlink channel training use, therefore we need at least length- $M$ channel training sequences to obtain a meaningful estimation, otherwise we would end up with underdetermined linearequations. The extra training symbols beyond length- $M$ is used to refine the estimation from channel noise. Similar properties also present themselves in the CSIT feedback process. On the other hand, in a spatially correlated channels, suppose that the effective rank of $\boldsymbol{R}_{n}$ is $r_{n}$, it is straightforwardly to apply the analysis above to conclude that the dimensionality loss scales with $r_{n}$. In our previous work [6], an efficient channel training and feedback scheme has been proposed with dimensionality loss that scales well with the effective rank of the CCM. Yet the connection between the dimensionality loss scaling factor and the physical channel properties, mainly the APS in this paper, has not been properly illustrated, which leads to our main results.

\section{A. Main Results}

Theorem 1: Consider a uniform linear antenna-array with length $L$, carrier wave length $\lambda$ and without loss of generality, assuming antenna spacing $D=\frac{\lambda}{2},{ }^{3}$ for an $M$-dimensional downlink channel vector $\boldsymbol{h}$, with $\boldsymbol{h} \sim \mathcal{C N}(\mathbf{0}, \boldsymbol{R})$, the rank of $\boldsymbol{R}$, i.e., the dimensionality loss scaling factor, is

$$
r \stackrel{M \rightarrow \infty}{\longrightarrow} \frac{L}{\lambda} \Omega
$$

where $\Omega$ is defined as the angular spread in terms of directional cosines.

Proof: See Appendix A.

Remark 1: We stress that in real systems, Theorem 1 may be approximately satisfied, because the direction-of-departure (DoD) is never perfectly confined in an angular spread. In fact, it is shown that a Laplacian distribution matches the real APS measurements closely [7]. Therefore, an effective angular spread is needed for general APSs in Theorem 1, which will be discussed in Section III. Additionally, the large system asymptotic introduces some approximations, such as nonignorable side lobes when a finite $M$ is considered. Despite

\footnotetext{
${ }^{3}$ Notice that this is the so-called critically placed antennas, and it is wellknown that placing the antennas more densely will not increase angular resolvability.
} 
of the approximations, Theorem 1 gives an insightful and informative measurement of the dimensionality loss in FDD massive MIMO downlinks.

Remark 2: Notice that the full span of directional cosine $\Omega$ is 2 , and in this circumstance,

$$
r \stackrel{M \rightarrow \infty}{\longrightarrow} \frac{2 L}{\lambda}=M
$$

which means the dimensionality loss scales with $M$. Therefore, the conventional belief of i.i.d. channels where the dimensionality loss scales with $M$ corresponds to the scenario where the user DoD spans the entire angular domain ${ }^{4}$.

Corollary 1: The number of downlink transmit diversity, which is defined as the number of non-zero columns in the angular representation of the channel matrix $\boldsymbol{H}$, is expressed as

$$
\mathcal{D}_{\mathrm{T}} \stackrel{M \rightarrow \infty}{\longrightarrow} \frac{L}{\lambda}\left(\bigcup_{n} \Omega_{n}\right),
$$

where $\Omega_{n}$ denotes the angular spread of the $n$-th user, in terms of directional cosines.

Proof: See Appendix B.

Remark 3: It is proved in [4, Theorem 3] that when the angular-spread intervals of the users are disjoint, the supports of their channel vectors in singular vector space are orthogonal. Then the total transmit diversity is the sum of the ranks of CCMs for orthogonal linear space. While Corollary 1 generalizes the results to the scenario where user angular-spread intervals are overlapped, and more importantly, Corollary 1 is more insightful since it connects the transmit diversity with the angular representation of the channels shown in Appendix B.

Remark 4: It is enlightening to discuss the tradeoff between the dimensionality loss and the downlink transmit diversity based on Theorem 1 and Corollary 1. Notice that the D$\mathrm{oF}$ of the downlink broadcast channel can be expressed as $\min \left(\mathcal{D}_{\mathrm{T}}, N\right)$. Theorem 1 gives the dimensionality loss scaling factor, which can be much less than the conventional belief of $M$, however, the transmit diversity is not necessarily reduced as long as the user DoDs spread over the entire angular domain. In other words, it is possible to estimate a full-rank channel matrix with a much smaller dimensionality loss.

\section{Simulations}

In this section, we use a 2-dimensional GSCM to simulate a simplified ray tracing of wireless signal propagations [8]. We adopt a single-scattering model, as shown in Fig. 1, where we only consider the MPCs which go through singlescattering specular process (BS to scatters to user) and the line-of-sight (LoS) MPC. The scatters are located according to a given spatial distribution. The reason we only consider single-scattering instead of multiple-scattering is that it is proved that there exists a unique transformation between the location of scatters (in a single-scattering model) and the

\footnotetext{
${ }^{4}$ Notice that since $\Omega$ is directional cosine, the angular spread only needs to be $\pi$ to cover the entire domain.
}

TABLE I

SYSTEM PARAMETERS

\begin{tabular}{lr}
\hline Carrier frequency $f_{c}$ & $3.5 \mathrm{GHz}$ \\
Number of BS antennas & 200 \\
Number of user antennas & 1 \\
Distance from BS to user & $500 \mathrm{~m}$ \\
User mean DoD & $\frac{\pi}{2}$ \\
Scatters location distributions & Uniform in a circle around each \\
& user with radius $=100 \mathrm{~m}$. \\
Antenna spacing & $\frac{\lambda}{2}$ \\
Number of scatters & 100 \\
Power attenuation at scattering & $-2.2 \mathrm{~dB}$ \\
Simulation iterations & 200 \\
\hline
\end{tabular}

delay and azimuth of the signals seen at BS side [9]. In other words, there always exists an equivalent single-scattering model, whose delay and azimuth are exactly the same with the actual multiple-scattering process. We emphasize that to give a comprehensive simulation of the real signal propagation, there are several other phenomenons which actually take effect, such as far scatters, wave-guiding, polarization, diffuse and diffraction. These effects may result in a larger angular spread and change the APS, however since we are considering the double-directional channel given the angular spread, it is sufficient and computationally efficient to adopt a singlescattering model.

The amplitude of a MPC is given by

$$
a_{i}=\frac{1}{\sqrt{p_{\mathrm{S}}} \frac{4 \pi d_{i}}{\lambda}},
$$

where $p_{\mathrm{S}}$ denotes the power attenuation at the scatter, $d=$ $\left(d_{\text {UE-SCT }}+d_{\text {SCT-BS }}\right)$ for single scattering MPC, $d=d_{\text {UE-BS }}$ for LoS MPC, $d_{\mathrm{UE}-\mathrm{SCT}}$ denotes the distance between the user and the scatterer and etc. The phase shift along the path is

$$
\phi_{i}=2 \pi d_{i} / \lambda
$$

and the phase shift introduced by specular scattering is modeled by a uniform distribution. The specific values of parameters used in the simulations are listed in Table I.

The singular value distribution is shown in Fig. 2, where the singular values are computed according to the one-ring model,

$$
[\boldsymbol{R}]_{i, j}=\frac{1}{2 \Delta} \int_{-\Delta+\theta}^{\Delta+\theta} e^{-j 2 \pi D(i-j) \cos (\alpha)} d \alpha,
$$

the Laplacian model,

$$
[\boldsymbol{R}]_{i, j}=\frac{1}{\sqrt{2} \Delta} \int_{\theta-\pi}^{\theta+\pi} e^{-\frac{\sqrt{2}}{\Delta}|\alpha-\theta|-j 2 \pi D(i-j) \cos (\alpha)} d \alpha .
$$

and the GSCM. The singular values are depicted in descending order, and the index is the $x$-axis. The theoretical rank of the CCM is computed according to Theorem 1. It is shown that Theorem 1 is very accurate for the GSCM and the one-ring model, based on the observation that singular values beyond the threshold is quite small. However, it is also observed that Theorem 1 is overly optimistic about the dimensionality loss 


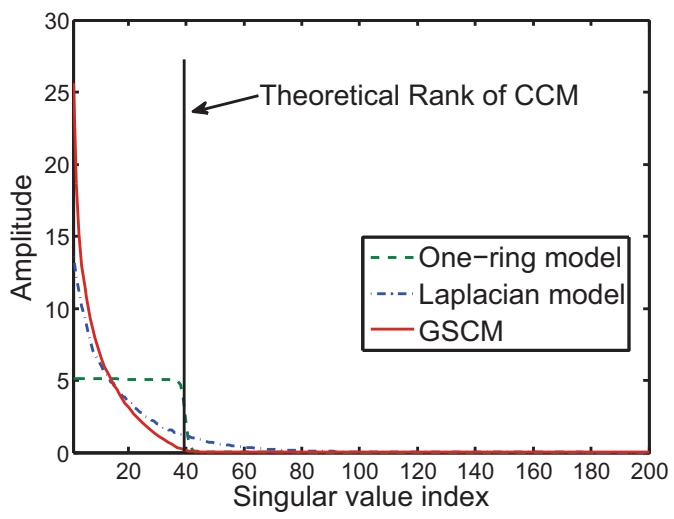

Fig. 2. Sorted singular values which are computed according to the one-ring model, Laplacian distribution and the GSCM. The scatters in the GSCM are located uniformly in a circle around the user.

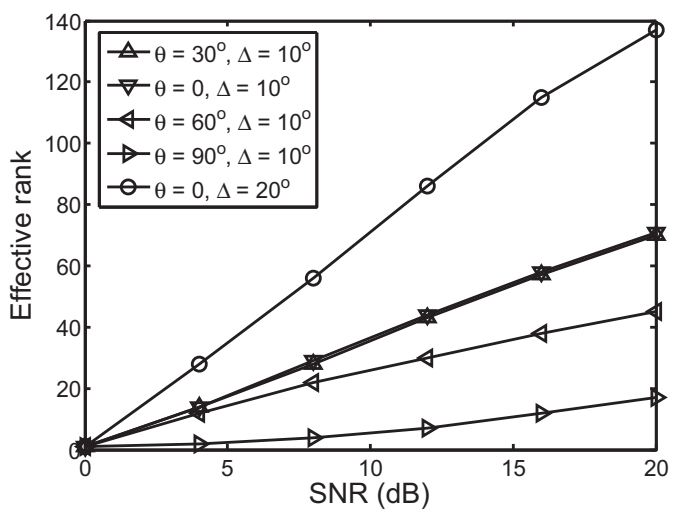

Fig. 3. The effective rank of the CCM which is computed according to the Laplacian distribution (11). $M=200$

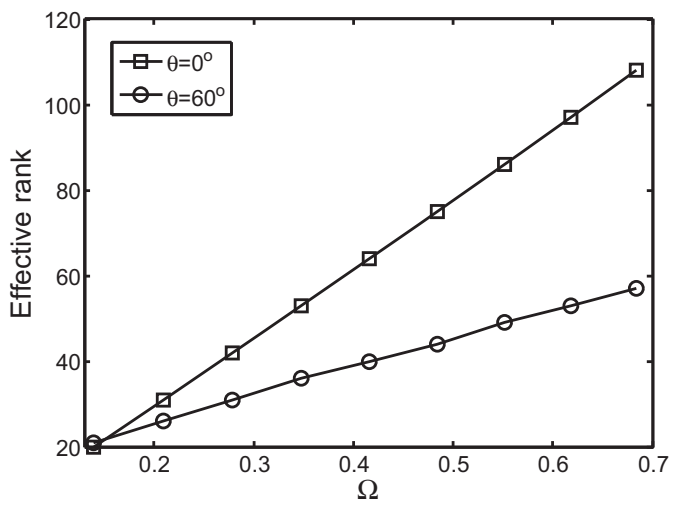

Fig. 4. The effective rank of the CCM which is computed according to the Laplacian distribution (11), versus the directional cosine span. The downlink SNR is 15 dB. $M=200$

when using the Laplacian model, for the reason that in the onering model or the GSCM, the angular spread is restricted in the given interval, whereas in the Laplacian model, considerable amount of angular power is "spilled over" outside the interval $[-\Delta+\theta, \Delta+\theta]$.

In order to study the dimensionality loss scaling factor with a Laplacian APS, we give numerical results on the effective rank of the CCM, given the transmit signal-to-noise ratio (SNR). The effective rank, $r_{\text {eff }}$ is assumed to be the $r_{\text {eff }}$ largest singular values, such that the power of the $M-r_{\text {eff }}$ remaining singular values is smaller than $1 / \mathrm{SNR}$, i.e., the power of the neglected singular values vanishes below the considered noise level. The relationship between the effective rank and the transmit SNR is shown in Fig. 3, with various mean DoDs and angular spreads. It is observed that the effective rank generally increases with transmit $\mathrm{SNR}^{5}$, due to the fact that the larger the SNR is, the more singular values we should estimate to guarantee the neglected power is below the noise level. Additionally, the effective rank also decreases as the DoD becomes smaller. This can be explained by Theorem 1 which stated the rank of the CCM is linear with the directional cosine span of the signal. Therefore, as the DoD becomes smaller, given the same angular spread $\Delta$, the effective rank is smaller because the directional cosine covers smaller interval. It is also very intuitive to observe that the effective rank increases with the angular spread $\Delta$.

Remark that since generally the angular power is never perfectly confined in a given interval, such that the condition in Theorem 1 is not met exactly, however, Theorem 1 is still very valuable in the sense that it serves as an instructional measure of the dimensionality loss scaling factor in the system design.

The linearity of the effective rank with the directional cosine span is validated in Fig. 4. It is shown that although the angular power is not confined by using the Laplacian model, the linear relationship, which is stated in Theorem 1 assuming restricted angular power, still holds. The cosine span in the figure is obtained by changing the angular spread of the signal.

\section{CONCLUSIONS}

It is found that by assuming perfect knowledge of the CCMs at both sides of the downlink broadcast channel, the dimensionality loss scaling factor for a single user is $\frac{L}{\lambda} \Omega$ in the large system limit of FDD massive MIMO systems, where $\Omega$ is the directional cosine span. The number of transmit diversity at the BS side is $\frac{L}{\lambda}\left(\bigcup_{n} \Omega_{n}\right)$. The scaling results are very helpful to understand the downlink channel estimation overhead in nonreciprocal systems, and to optimize the system throughput. Simplified ray tracing simulations validate our results, and show that when angular power is not perfectly confined, the linear relationship still holds, by computing the effective rank of the CCM using the Laplacian model.

\section{ACKNOWLEDGEMENT}

This work is sponsored in part by the National Basic Research Program of China (973 Program: 2012CB316001), the National Science Foundation of China (NSFC) under grant No. 61201191 and No. 61321061, and Hitachi R\&D Headquarter. The authors would like to thank Dr. A. Molisch and Dr. G. Caire for inspiring discussions.

\footnotetext{
${ }^{5}$ Notice that the effective rank will eventually saturate if SNR further increases to the point when all singular values are relevant, and the SNR range we show in Fig. 3 is practical in current cellular systems.
} 


\section{APPENDIX A \\ PROOF OF THEOREM 1}

Proof: Consider the transformation from the deterministic double-directional channel to the vector channel [10],

$$
\boldsymbol{h}=\sum_{i=1}^{B} a_{i} e^{j \phi_{i}} \boldsymbol{e}\left(\cos \left(\theta_{i}\right)\right),
$$

where the summation is over all $B$ significant multi-path components (MPCs), $a_{i}$ is the receive amplitude of the $i$-th MPC, $\phi_{i}$ is the phase shift along the transmit path, and $\boldsymbol{e}\left(\cos \left(\theta_{i}\right)\right)$ is defined as the unit spatial signature with direction-of-departure (DoD) $\theta_{i}$

$$
\begin{aligned}
\boldsymbol{e}\left(\cos \left(\theta_{i}\right)\right)= & \frac{1}{\sqrt{M}}\left[1, \exp \left(-\frac{j 2 \pi D \cos \left(\theta_{i}\right)}{\lambda}\right), \ldots,\right. \\
& \left.\exp \left(-\frac{j 2 \pi(M-1) D \cos \left(\theta_{i}\right)}{\lambda}\right)\right]^{\dagger} .
\end{aligned}
$$

The angular representation of $\boldsymbol{h}$, whose orthonormal basis corresponds to the angular resolvable bins shown in Fig. 1, is expressed as [11]

$$
\boldsymbol{h}^{\mathrm{a}}=\boldsymbol{U}^{\dagger} \boldsymbol{h}
$$

where

$$
\boldsymbol{U}=\left[\boldsymbol{e}(0), \boldsymbol{e}\left(\frac{1}{L / \lambda}\right), \ldots, \boldsymbol{e}\left(\frac{M-1}{L / \lambda}\right)\right]
$$

Then,

$$
\begin{aligned}
h_{m}^{\mathrm{a}} & =\sum_{i=1}^{B} a_{i} e^{j \phi} \omega_{m, i}, \\
\omega_{m, i} & =\boldsymbol{e}^{\dagger}\left(\frac{m-1}{L / \lambda}\right) \boldsymbol{e}\left(\cos \left(\theta_{i}\right)\right),
\end{aligned}
$$

and

$$
\omega_{m, i}=\frac{1}{M} \exp \left(j \pi(M-1) D C_{m, i} / \lambda\right) \frac{\sin \left(\pi L C_{m, i} / \lambda\right)}{\sin \left(\pi L C_{m, i} /(M \lambda)\right)},
$$

where $C_{m, i}=\frac{m-1}{L / \lambda}-\cos \left(\theta_{i}\right)$. For

$$
\left|C_{m, i}\right|>\frac{\sqrt{M} \lambda}{L}=\frac{1}{\sqrt{M} D}
$$

it follows that,

$$
\begin{aligned}
\left|\omega_{m, i}\right| & \leq \frac{1}{M\left|\sin \left(\pi L C_{m, i} /(M \lambda)\right)\right|} \\
& <\frac{1}{M \sin (\pi / \sqrt{M})} \\
& \stackrel{M \rightarrow \infty}{\longrightarrow} 0 .
\end{aligned}
$$

Combining (18) and (19), it is proved that in the large system limit, the $m$-th component of the angular representation, $h_{m}^{\mathrm{a}}$ is non-zero, only if $\frac{m-1}{L / \lambda}$ is within the angular spread of the MPC directional cosines, up to a constant representing the side lobes shown in (18). Therefore, given the interval between the base vectors $U$ as $\frac{1}{L / \lambda}$, the number of non-zero components in $\boldsymbol{h}^{\mathrm{a}}$, denoted by $\eta$, is

$$
\eta=\frac{\Omega}{\frac{1}{L / \lambda}}+\frac{2}{\sqrt{M} D} \stackrel{M \rightarrow \infty}{\longrightarrow} \frac{L}{\lambda} \Omega .
$$

Notice that $\boldsymbol{U}$ is a discrete-Fourier-transformation (DFT) matrix, which coincides with the singular matrix of the CCM, $\boldsymbol{R}$ when $M$ is large [4]. Therefore, the rank of $\boldsymbol{R}$ equals $\eta$, which concludes the proof.

\section{APPENDIX B}

PROOF OF COROLLARY 1

Consider the angular representation of the downlink channel matrix $\boldsymbol{H}$,

$$
\boldsymbol{H}^{\mathrm{a}}=\boldsymbol{H} \boldsymbol{U}=\left[\boldsymbol{h}_{1}^{\mathrm{a}}, \boldsymbol{h}_{2}^{\mathrm{a}}, \ldots, \boldsymbol{h}_{N}^{\mathrm{a}}\right]^{\dagger} .
$$

Notice that the $i$-th column of $\boldsymbol{H}^{\text {a }}$ is non-empty iff there exists one user with angular spread measured in directional cosine overlapping with $\frac{i-1}{L / \lambda}$, given by Theorem 1 . Therefore, the total number of non-empty columns is the union of the angular spread of all users measured in directional cosine, up to a multiplicative constant. This concludes the proof.

From a CCM standpoint, the transmit diversity can also be defined as the [4]

$$
\operatorname{dim}\left(\operatorname{Span}\left(\bigcup \boldsymbol{S}_{n}\right)\right), \forall n,
$$

where $\boldsymbol{S}_{n}$ is defined as the support of $\boldsymbol{h}_{n}$, i.e., the singular vector space corresponding to the non-zero singular values of the $\boldsymbol{R}_{n}$. Based on this definition, and Theorem 1 that in the large system limit, the singular matrices of all CCMs are a DFT matrix, i.e., $\boldsymbol{U}$ as in (15), the transmit diversity can be also expressed as the number of non-empty rows of

$$
\mathcal{S}=\left[\boldsymbol{U}^{\dagger} \boldsymbol{R}_{1} \boldsymbol{z}_{1}, \boldsymbol{U}^{\dagger} \boldsymbol{R}_{2} \boldsymbol{z}_{2}, \ldots, \boldsymbol{U}^{\dagger} \boldsymbol{R}_{N} \boldsymbol{z}_{N}\right]=\left(\boldsymbol{H}^{\mathrm{a}}\right)^{\dagger},
$$

which is equivalent with (21).

\section{REFERENCES}

[1] J. Andrews, S. Buzzi, W. Choi, S. Hanly, A. Lozano, A. Soong, and J. Zhang, "What will 5g be?," IEEE J. Sel. Areas Commun., vol. 32, pp. 1065-1082, Jun 2014.

[2] T. Marzetta, "Noncooperative cellular wireless with unlimited numbers of base station antennas," IEEE Trans. Wireless Commun., vol. 9, pp. 3590-3600, Nov 2010.

[3] H. Q. Ngo, E. Larsson, and T. Marzetta, "Energy and spectral efficiency of very large multiuser mimo systems," IEEE Trans Commun., vol. 61, pp. 1436-1449, Apr 2013.

[4] A. Adhikary, J. Nam, J.-Y. Ahn, and G. Caire, "Joint spatial division and multiplexing: The large-scale array regime," IEEE Trans. Inform. Theory, vol. 59, pp. 6441-6463, Oct 2013.

[5] A. Adhikary, E. Al Safadi, M. Samimi, R. Wang, G. Caire, T. Rappaport, and A. Molisch, "Joint spatial division and multiplexing for mm-wave channels," IEEE J. Select. Areas Commun., vol. 32, pp. 1239-1255, Jun 2014.

[6] Z. Jiang, A. F. Molisch, G. Caire, and Z. Niu, "Achievable rates of fdd massive mimo systems with spatial channel correlation," submitted to IEEE Trans. Wireless Comm., available online at arXiv:1406.7486.

[7] K. Pedersen, P. Mogensen, and B. Fleury, "Spatial channel characteristics in outdoor environments and their impact on base station antenna system performance," in IEEE VTC, vol. 2, pp. 719-723, 1998.

[8] A. Molisch, "A generic model for mimo wireless propagation channels in macro- and microcells," IEEE Trans. Signal Processing, vol. 52, pp. 6171, Jan 2004. 
[9] P. Petrus, J. Reed, and T. Rappaport, "Geometrical-based statistical macrocell channel model for mobile environments," IEEE Trans. Commum., vol. 50, pp. 495-502, Mar 2002.

[10] A. F. Molisch, Wireless communications, vol. 15. John Wiley \& Sons,
2010.

[11] D. Tse and P. Viswanath, Fundamentals of wireless communication. Cambridge university press, 2005. 